\title{
ON THE CLIFFORD COLLINEATION, TRANSFORM AND SIMILARITY GROUPS (IV) AN APPLICATION TO QUADRATIC FORMS
}

\author{
G. E. WALL
}

To RICHARD BRAUER on his 60th birthday

\section{Introduction}

E. S. Barnes and I recently ${ }^{1)}$ constructed a series of positive quadratic forms $f_{N}$ in $N=2^{n}$ variables $(n=1,2, \ldots)$ with relative minima of order $N^{\frac{1}{2}}$ for large $N$. I continue this investigation by determining the minimal vectors of $f_{N}$ and showing that, for $N \neq 8$, its group of automorphs is the Clifford group ${ }^{2)}$ $\mathscr{C} \mathscr{T}_{1}^{+}\left(2^{n}\right)(\S 3)$. This suggests a generalization. Replacing $\mathscr{C} \mathscr{T}_{1}^{+}\left(2^{n}\right)$ by $\mathscr{C} \mathscr{T}\left(p^{n}\right)$, where $p$ is an odd prime, I derive a new series of positive forms in $N=(p-1) p^{n}$ variables $(\S 4)$. The relative minima are again of order $N^{\frac{1}{2}}(p$ fixed, $N \rightarrow \infty$ ), the "best" forms being those for $p=3,5$. All forms are eutactic though only those for $p=3,5$ are extreme.

The methods used here raise several questions. Firstly, the forms constructed have fairly big relative minima while the representations of the symplectic group $S p(2 n, p)$ associated with $\mathscr{C} \mathscr{T}\left(p^{n}\right)$ are of smallest possible degree (CGI, theorem 10). Are these two facts directly related? Secondly, it is natural to regard the lattice introduced in $\S 4.2$ as a commutative algebra. Is there a simple direct relation between this algebra and the automorph group $\mathscr{C} \mathscr{T}\left(p^{n}\right)$ ?

\section{Preliminaries}

The notation used in this paper is a compromise between that of EF and that of CGI, CGII. See in particular $\S 2.1-2.3$ below.

\subsection{Vector spaces and groups over $G F(p)$.}

Throughout this paper, $p$ stands for a fixed prime and $n$ for a fixed natural

Received Nov. 22, 1961.

1) Cf. [1]. This paper is referred to as EF.

2) Cf. [2], [3]. These papers are referred to as CGI, CGII. 
number. $\quad V=V_{n}(p)$ denotes the vector space of all row vectors $\alpha=\left(\alpha_{1}, \ldots\right.$ $\alpha_{n}$ ) over the Galois field $G F(p) . \quad V_{r}$ stands generically for an $r$-dimensional subspace of $V, C_{r}$ for a coset $\alpha+V_{r}$.

It is easily proved that each function $f(\alpha)$ defined on $V$ and with values in $G F(p)$ coincides in value with a unique polynomial $P\left(\alpha_{1}, \ldots, \alpha_{n}\right)$ of degree $<p$ in each $\alpha_{i}$. Such polynomials will be called standard. The degree of $f$ is defined as the total degree of $P$.

Let $p=2$. Consider the $2 n$-dimensional quadratic form $\phi(\lambda)=\sum_{1}^{n} \lambda_{i} \lambda_{n+i}$ over $G F(2)$, where $\lambda$ is the row vector $\left(\lambda_{i}\right)(i=1, \ldots, 2 n)$. The ( $2 n$-rowed) matrices $^{3)} T$ which leave $\phi(\lambda)$ invariant, i.e., $\phi(\lambda)=\phi\left(\lambda T^{\prime}\right)$, form the orthogonal group $O_{1}(2 n, 2)$. Let

$$
\begin{aligned}
T & =\left(\begin{array}{ll}
P & Q \\
R & S
\end{array}\right) \quad(P, Q, R, S n \times n \text { matrices }) \\
d_{T} & =\operatorname{rank} R .
\end{aligned}
$$

The $T$ such that $d_{r}$ is even form the rotation subgroup $O_{1}^{+}(2 n, 2)$.

Let $p>2$. Consider the $2 n$-dimensional alternate bilinear form

$$
f(\lambda, \mu)=\sum_{1}^{n}\left(\lambda_{i} \mu_{n+i}-\mu_{i} \lambda_{n+i}\right)
$$

over $G F(p)$. The matrices $T$ which leave $f(\lambda, \mu)$ invariant, i.e., $f(\lambda, \mu)$ $=f\left(\lambda T^{\prime}, \mu T^{\prime}\right)$ form the symplectic group $S p(2 n, p)$. The notation (2.1.1) will also be used for the elements of $S p$.

\subsection{Vector spaces and groups over the cyclotomic field $P$.}

Let $R_{0}$ denote the rational field, $P$ the $p$-th cyclotomic field: $P=R_{0}(\omega)$, where $\omega=\exp (2 \pi i / p)$. Then $E=E_{p^{n}}$ denotes a $p^{n}$-dimensional vector space over $P$. We choose a fixed basis of $E$, indexing its $p^{n}$ members $\mathbf{e}_{\alpha}$ with the $p^{n}$ elements $\alpha$ of $V$. We use the notations

$$
\mathbf{x}=\left(x_{\alpha}\right)=\sum x_{\alpha} \mathbf{e}_{\alpha}
$$

for the elements of $E$.

The scalar product on $E$ is defined by

3) The transpose of a matrix $T$ in denoted by $T^{\prime}$. 


$$
\left(x_{\alpha}\right) \cdot\left(y_{\alpha}\right)=\sum_{\alpha \in V} \bar{x}_{\alpha} y_{\alpha}
$$

The terms unitary $(p>2)$, orthogonal $(p=2)$ are interpreted accordingly.

Let $p=2$. The Clifford transform group $\mathscr{C} \mathscr{T}_{1}^{+}\left(2^{n}\right)^{4)}$ is a group of orthogonal transformations on $E$. There exists a homomorphism of $\mathscr{C} \mathscr{T}_{1}^{+}\left(2^{n}\right)$ onto $O_{1}^{+}(2 n, 2)$ such that each original of $T \in O_{1}^{+}$has the form ${ }^{5)}$

$$
X \mathbf{e}_{a}=2^{-\frac{1}{2} d T} \sum_{\beta \in C}(-1)^{f(\beta)} \mathbf{e}_{\beta},
$$

where $C$ is a coset of dimension $d_{T}, f$ a function of degree $\leq 2$. For each function $g(\alpha)$ of degree $\leq 2$, non-singular $n \times n$ matrix $D$ over $G F(2)$ and vector $\mathbf{t} \in V$, the linear transformation ${ }^{6)}$

$$
Y \mathbf{e}_{\alpha}=(-1)^{g(\alpha)} \mathbf{e}_{\alpha D+\mathbf{t}}
$$

belongs to $\mathscr{C} \mathscr{T}_{1}^{+}$.

Let $p>2$. The Clifford transform group $C T\left(p^{n}\right)$ was defined in CGI $\S 3.1$. We define $\mathscr{C} \mathscr{T}\left(p^{n}\right)$ as the commutator group of $C T\left(p^{n}\right)$ when $p^{n}>3$, as the group $\{Y, Z\} \mathscr{C} \mathscr{G}$ in CGI Appendix, section (4), when $p^{n}=3 . \mathscr{C} \mathscr{T}\left(p^{n}\right)$ is a group of unitary transformations on $E$. There exists a homomorphism of $\mathscr{C} \mathscr{T}\left(p^{n}\right)$ onto $\operatorname{Sp}(2 n, p)$ such that each original of $T \in S p$ has the form ${ }^{i}$

$$
X \mathbf{e}_{\alpha}= \pm \theta^{-d_{T}} \sum_{\beta \in C} \omega^{f(\boldsymbol{\beta})} \mathbf{e}_{\boldsymbol{\beta}},
$$

where $C$ is a coset of dimension $d_{T}, f$ a function of degree $\leq 2$ and

$$
\theta=\sum_{i=0}^{p-1} \omega^{i^{2}}
$$

For each function $g(\alpha)$ of degree $\leq 2$, non-singular $n \times \boldsymbol{n}$ matrix $D$ over $G F(p)$ and vector $\mathrm{t} \in V$, the linear transformation ${ }^{8)}$

$$
Y \mathbf{e}_{\alpha}=\omega^{g(\alpha)} \mathbf{e}_{\alpha D+\mathbf{t}}
$$

belongs to $\mathscr{C} \mathscr{T}$.

1) Defined in CGII $\S 3.3$, for $n \geq 3$ only, as the commutator group of $C T\left(2^{n}\right)$. A universal definition is that $\mathscr{C} \mathscr{T}_{1}^{+}\left(2^{n}\right)$ consists of the elements in CGII $(5.10)$ corresponding to the elements $T$ of $O_{1}^{+}(2 n, 2)$.

5) See CGII (510) and (5.5).

6) These are the elements in CGII (5.10) corresponding to $d_{T}=0$.

7) See CGI (3.1.1) and (4.1.6).

8) These are the elements of $\mathscr{C} \mathscr{T}$ corresponding to the $T$ with $d_{T}=0$. 
2.3. Lattices. Let $\Omega$ denote the ring of all integers in $P$. We define an $\Omega$ lattice as the set of all integral linear combinations

$$
\sum \xi_{\alpha} \mathbf{u}_{\alpha} \quad\left(\xi_{\alpha} \in \Omega\right)
$$

of $p^{n}$ linearly independent vectors $\mathbf{u}_{\alpha}$. In particular, $l=\Gamma_{p^{n}}$ denotes the $\Omega$ lattice of all integral vectors

$$
\sum x_{a} \mathbf{e}_{\alpha} \quad\left(x_{\alpha} \in \Omega\right) .
$$

If $\Lambda_{1}, \Lambda_{2}$ are $\Omega$-lattices and $\Lambda_{1} \subset \Lambda_{2}$, the grouptheoretical index $\left|\Lambda_{2}: \Lambda_{1}\right|$ is finite. In particular, if $\lambda(\neq 0) \in \Omega$, we have

$$
|\Gamma: \lambda \Gamma|=|N(\lambda)|^{p^{n}}
$$

where $N(\lambda)$ is the norm of $\lambda$ in $P$ relative to $R_{0}$. For, if $\mathbf{x}, \mathbf{y} \in \Gamma$ then $\mathbf{x} \equiv \mathbf{y}$ $(\bmod \lambda \Gamma)$ if, and only if, $x_{\alpha} \equiv y_{\alpha}(\bmod \lambda)$ for each $\alpha \in V$.

Let $A$ be an $\Omega$-lattice such that $\lambda I^{\prime} \subset \Lambda \subset \Gamma$, where $\lambda(\neq 0) \in \Omega$. We define the dual $\Lambda^{\prime}$ of $\Lambda$ modulo $\lambda$ as follows: $\Lambda^{\prime}$ is the set of $\mathbf{x} \in E$ such that $\mathbf{x} \cdot \mathbf{y} \in \lambda \Omega$ for all $\mathbf{y} \in A$. Since $\lambda \Gamma \subset A \subset I$, we have $\bar{\lambda} \Gamma \subset A^{\prime} \subset \Gamma$. The argument of EF $\S 2$ shows that $\Lambda^{\prime}$ is an $\Omega$-lattice, that $\Lambda$ is the dual of $\Lambda^{\prime}$ modulo $\bar{\lambda}$ and that

$$
|\Gamma: \Lambda|\left|\Gamma: \Lambda^{\prime}\right|=\left|\Gamma: \lambda I^{\prime}\right|=\left.N(\lambda)\right|^{p^{n}} .
$$

It is a well known theorem that every finitely generated module over a principal ideal ring has a basis. The following variant is proved in exactly the same way.

Lemma 2.3.1. Let $\lambda(\neq 0) \in \Omega$ and suppose that every divisor of the principal ideal with generator $\lambda$ is principal. Then every $\Omega$-module $M$ such that $\lambda \Gamma \subset M \subset \Gamma$ is an $\Omega$-lattice.

\subsection{Criteria for quadratic functions on $V_{n}(2)$.}

In the present section, $p=2$ and $f(\alpha)$ is a function defined on $V$ with values in $G F(2)$. If $W \subset V$, we write

$$
\langle W ; f\rangle=\sum_{a \in W^{\prime}}(-1)^{f(a)} .
$$

As stated in $\S 2.1 ., f$ has a unique expression in the form

$$
f(\alpha)=\sum_{s} a_{s} \alpha_{s} \quad\left(a_{s} \in G F(2), \alpha_{s}=\prod_{i \in s} \alpha_{i}\right),
$$

where summation is over the subsets (including the empty set) of $1,2, \ldots, n$. . 
We note that, since $\alpha^{2}=\alpha$ on $G F(2)$, the degree of $f(\alpha)$ is $\leq 2$ if, and only if, the function $g(\alpha)=f(\alpha)+f(0)$ is a quadratic form.

LEMMA 2.4.1. The degree of $f(\alpha)$ is $\leq 2$ if, and only if, $f(\alpha)$ has an even number of zeros in every $V_{3} \subset V$.

Proof. The following conditions for a scalar-valued function $h(\mathbf{u})$ on a vector space to be a quadratic form are well known:

(i) the function $k(\mathbf{u}, \mathbf{v})=h(\mathbf{u}+\mathbf{v})-h(\mathbf{u})-h(\mathbf{v})$ is bilinear, and

(ii) $h(\lambda \mathbf{u})=\lambda^{2} h(\mathbf{u})$.

It follows that $h$ is a quadratic form if, and only if, its restriction to every subspace of dimension $\leq 3$ is a quadratic form. It is therefore sufficient to prove our lemma for $n \leq 3$. For $n \leq 2$, the lemma is obvious from (2.4.2). For $n=3$, it follows from the formula $\sum_{\alpha \in V} f(\alpha)=a_{(1,2,3)}$.

Corollary. The degree of $f(\alpha)$ is $\leq 2$ if, and only if,

$$
\left\langle V_{3} ; f\right\rangle \equiv 0(\bmod 4) \text { for every } V_{3} \subset V .
$$

We suppose from now on that the degree of $f(\alpha)$ is $\leq 2$. Let $q(\alpha)=f(\alpha)$ $+f(0)$ be the corresponding quadratic form. The polar form of $q$ is the bilinear form

$$
Q(\alpha, \beta)=q(\alpha+\beta)+q(\alpha)+q(\beta) .
$$

Since $Q$ is alternate $(Q(\alpha, \alpha) \equiv 0)$ its rank is even, say $2 d$. We call $d$ the reduced rank of $f$.

LemMa 2.4.2. Suppose that $f(\alpha)$ has degree $\leq 2$, reduced rank $\leq D$. Then, for each $V_{k} \subset V(0 \leq k \leq n)$,

$$
\left\langle V_{k} ; f\right\rangle \equiv 0\left(\bmod 2^{t}\right),
$$

$$
\text { where }^{9)} t=\max \left(\left[\frac{1}{2}(k+1)\right], k-D\right) .
$$

Proof. Let $d$ be the reduced rank of $f$. Then $q(\alpha)=f(\alpha)+f(0)$ is equivalent ${ }^{10 \prime}$ to one of

$$
q_{1}(\alpha)=\sum_{1}^{d} \alpha_{i} \alpha_{d+i}, \quad q_{2}(\alpha)=q_{1}(\alpha)+\alpha_{1}+\alpha_{d+1}, \quad q_{3}(\alpha)=q_{1}(\alpha)+\alpha_{2} d+1 .
$$

\footnotetext{
9) $[r]=$ integral part of $r$.

10) See e.g., Dieudonné [6].
} 
The number of zeros of $q(\alpha)$ is accordingly

$$
2^{n-1}+e 2^{n-d-1} \quad(\varepsilon=1,-1 \text { or } 0)
$$

and so $\langle V ; f\rangle=\varepsilon 2^{n-d}$. Therefore

$$
\langle V ; f\rangle \equiv 0\left(\bmod 2^{n-d}\right)
$$

and, since $d \leq \frac{1}{2} n$,

$$
\langle V ; f\rangle \equiv 0\left(\bmod 2^{\left[\frac{1}{2}(n+1)\right]}\right) .
$$

Applying the last two congruences to the restriction $\bar{f}$ of $f$ to $V_{k}$ and noting that the reduced rank of $\bar{f}$ cannot exceed that of $f$ we get the lemma.

Lemma 2.4.3. Suppose that $f(\alpha)$ has degree $\leq 2$, reduced rank $d$. Let $D$ be an integer such that $0 \leq D \leq \frac{1}{2} n$. If

$$
\left\langle V_{2 D+2} ; f\right\rangle \equiv 0\left(\bmod 2^{D+2}\right) \text { for every } V_{2 D+2} \subset V \text {, }
$$

then $d \leq D$.

In fact, if $d$ were $>D$, the restriction of $q(\alpha)$ to a suitable $V_{2 D+2}$ would be equivalent to $\sum_{1}^{D+1} \alpha_{i} \alpha_{D+1+i}$; but then $\left\langle V_{2 D+2} ; f\right\rangle= \pm 2^{D+1}$, contrary to (2.4.5).

We shall later have to consider functions $h(\alpha)$ defined on a coset $\mathbf{a}+V_{k}$ rather than the whole of $V$. The degree and reduced rank of $h$ are defined to be those of the function $l(\beta)=h(\mathbf{a}+\beta)$, whose domain of definition is the subspace $V_{k}$.

\section{Lattices of dimension $2^{n}$}

We suppose throughout this section that $p=2$. The minimal vectors of the lattices $A(\lambda)$ are determined in $\S 3.1$, the automorphs of the "principal" lattices $A^{(1)}, A^{(2)}$ in $\S 3.2$.

We recall the definition of $A(\lambda)(\mathrm{EF} \S 3), \quad(\lambda)=\left(\lambda_{0}, \ldots, \lambda_{n}\right)$ is a set of integral indices satisfying

$$
\lambda_{0}=0, \lambda_{r}-1 \leq \lambda_{r-1} \leq \lambda_{r} \text { for } 1 \leq r \leq n,
$$

and $A(\lambda)$ is the lattice formed by all integral linear combinations of the vectors

$$
2^{\lambda_{n-r}}\left[C_{r}\right]=2^{\lambda_{n-r}} \sum_{\boldsymbol{a} \in C_{r}} \mathbf{e}_{\alpha},
$$

where $C_{r}$ runs over all cosets in $V$. 
If $W \subset V$ and $f(\alpha)$ is a function defined on $W$ with values in $G F(2)$, we write

$$
[W ; f]=\sum_{\alpha \in W}(-1)^{f(\alpha)} \mathbf{e}_{\alpha} .
$$

3.1. Minimal vectors of $\Lambda(\lambda)$. Let $\mathbf{x}$ be a minimal vector of $\Lambda=\Lambda(\lambda)$. By theorem 3.2. of EF,

$$
\mathbf{x}^{2}=2^{m}, \text { where } m=\min \left(n-r+2 \lambda_{r}\right),
$$

and $\mathbf{x}$ has the form

$$
\mathbf{x}=2^{\lambda R}[W ; f]
$$

where $R$ satisfies

$$
n-R+2 \lambda_{R}=m, \quad 0 \leq R \leq n,
$$

and $W$ is a subset of $V$ with $2^{n-R}$ elements.

We now complete this partial characterization by giving the conditions that a vector of the form (3.1.2) belong to $A$.

TheoRem 3.1. Let $R$ be an integer satisfying (3.1.3), $W$ a subset of $V$ with $2^{n-R}$ etements, $f(\alpha)$ a function defined on $W$ with values in $G F(2)$. Let $d$ be the largest integer such that

$$
\lambda_{R+k}=\lambda_{R}+\left[\frac{1}{2}(k+1)\right] \quad \text { for } \quad 0 \leq k \leq 2 d
$$

Then the vector $2^{\lambda}[W ; f] \in \Lambda(\lambda)$ if, and only if,

(i) $W$ is a coset $C_{n-R}$, and

(ii) $f(\alpha)$ has degree $\leq 2$, reduced rank $\leq d$.

Proof. Lemma 3.3 of EF can be sharpened by adding the following conditions for equality:

If precisely $2^{n-s}$ coordinates $x_{a}$ are odd, the corresponding a form a coset $C_{n-s}$

The proof is straightforward and is omitted. This sharper form of the lemma shows that (i) is a necessary condition.

We may now suppose that $W$ is a coset, or even a subspace $V_{n-R}$, because 
the results for cosets can easily be deduced by translation of coordinates. Now, each $V_{k} \subset V_{n-R}$ is the meet of $V_{n-R}$ with some $V_{k+R} \subset V$ but not the meet of $V_{n-R}$ with any $V_{k+R+u}, u>0$. Therefore, by theorem 3.1 of EF, the vector $\mathbf{x}=2^{\lambda R}[W ; f] \in \Lambda$ if, and only if,

$$
\left\langle V_{k} ; f\right\rangle \equiv 0\left(\bmod 2^{\mu_{k}}\right) \text { for every } V_{k} \subset \mathrm{V}_{n-R},
$$

where $\mu_{k}=\lambda_{R+k}-\lambda_{R}$. We remark that, by (3.1.4),

$$
\mu_{k}=\left[\frac{1}{2}(k+1)\right] \text { for } 0 \leq k \leq 2 d,
$$

and that, by (3.0.1) and (3.1.1),

$$
\mu_{2} d+2=d+2 \text { if } 2 d+2 \leq n-R,
$$

$$
\left[\frac{1}{2}(k+1)\right] \leq \mu_{k} \leq k-d \quad \text { for } \quad 2 d \leq k \leq n-R .
$$

Suppose now that $\mathrm{x} \in \Lambda$. By (3.1.5), (3.1.6) and (3.1.8),

$$
\left\langle V_{3} ; f\right\rangle \equiv 0(\bmod 4) \text { for every } V_{3} \subset V_{n-R},
$$

so that, by the corollary to lemma 2.4.1, the degree of $f \leq 2$. Again, by (3.1.5) and (3.1.7),

$$
\left\langle V_{2 d+2} ; \hat{f}\right\rangle \equiv 0\left(\bmod 2^{d+2}\right) \text { for every } V_{2 d+2} \subset V_{n-R},
$$

so that, by lemma 2.4 .3 , the reduced rank of $f \leq d$.

Conversely, suppose that $f$ has degree $\leq 2$, reduced rank $\leq d$. If $k \leq 2 d$, (3.1.5) holds by lemma 2.4.2 and (3.1.6). If $k>2 d,(3.1 .5)$ holds by lemma 2.4.2 and (3.1.8). Hence $x \in A$. This proves our theorem.

A straightforward enumeration of the quadratic functions of given reduced rank yields the total number of minimal vectors of rank $R$ stated in (5.10) of EF.

3.2. Automorphs of the principal lattices. The first and second principal lattices $\Lambda^{(1)}, \Lambda^{(2)}$ of dimension $N=2^{n}$ are the $\Lambda(\lambda)$ given by

$$
\lambda_{r}=\left[\frac{1}{2} r\right] \text { and }\left[\frac{1}{2}(r+1)\right](0 \leq r \leq n)
$$

respectively. They occupy a special position in that their (common) relative minimum $\left(\frac{1}{2} N\right)^{\frac{1}{2}}$ exceeds that of any other $A(\lambda)$ of dimension $N$. 
The fact that $\Lambda^{(1)}, \Lambda^{(2)}$ are dual modulo $2^{n}$ implies that they have the same group of automorphs. Suppose e.g. that $X$ is an automorph of $A^{(1)}$. If $\mathrm{x} \in \Lambda^{(1)}$, $\mathbf{y} \in \Lambda^{(2)}$, then

$$
\mathbf{x} \cdot X \mathbf{y}=X^{-1} \mathbf{x} \cdot \mathbf{y} \equiv 0\left(\bmod 2^{n}\right)
$$

Since this holds for all $\mathbf{x} \in \Lambda^{(1)}, X \mathbf{y} \in \Lambda^{(2)}$. Since $X \mathbf{y} \in \Lambda^{(2)}$ whenever $\mathbf{y} \in \Lambda^{(2)}$, $X$ is an automorph of $\Lambda^{(2)}$. The common group of automorphs is denoted by $\mathfrak{A}$.

By $\S 3.1$, the minimal vectors of $A^{(1)}$ ( $n$ odd $), A^{(2)}$ ( $n$ even) are

$$
2^{\left[\frac{1}{2} n\right]-s}\left[C_{2 s} ; f\right]
$$

where $C_{2 s}$ runs over all even-dimensional cosets in $V, f$ over all functions of degree $\leq 2$ on $C_{2 s}$; and those of $\Lambda^{(1)}$ ( $n$ even), $A^{(2)}$ ( $n$ odd) are

$$
2^{\left[\frac{1}{2}(n+1)\right]-s}\left[C_{2 s+1} ; f\right]
$$

where $C_{2 s+1}$ runs over all odd-dimensional cosets in $V, f$ over all functions of degree $\leq 2$ on $C_{2 s+1}$.

Theorem 3.2. If $n \neq 3, \mathfrak{A}=\mathscr{C} \mathscr{T}_{1}^{+}\left(2^{n}\right)$. If $n=3, \mathfrak{A} \cong\left[3^{4,2,1}\right]$ (in the notation of Coxeter and Moser [5]) and $\mathscr{C} \mathscr{T}_{1}^{+}\left(2^{3}\right)$ is a subgroup of $\mathfrak{A}$ of index 270.

Proof. Let $M_{s}$ denote the set of vectors (3.2.2) of fixed dimension $2 s, M$ the union of all the $M_{s}$. Write $\mathbf{u}_{9}=2^{\left[\frac{1}{2} n\right]}$ en. We first prove that

$$
M \text { is the set of all vectors } X \mathbf{u}_{3}\left(X \in \mathscr{C} \mathscr{T}_{1}^{+}\right) \text {. }
$$

By (2.2.1), $X \mathbf{u}, \in M$ if $X \in \mathscr{C} \mathscr{T}_{1}^{+}$. By (2.2.2) $\mathscr{C} \mathscr{T}_{1}^{+}$permutes the vectors in each $M_{s}$ transitively. It remains to prove that for each $s$ there is an $X \in \mathscr{C} \mathscr{T}_{1}^{+}$such that $X \mathbf{u}_{0} \in M_{s}$, i.e., by (2.2.1), that there is a $T \in O_{1}^{+}$such that $d_{T}=2 \mathrm{~s}$. The matrix $T$ defined as follows satisfies the requirement:

$$
\left.\begin{array}{ll}
\lambda \mathbf{T}^{\prime}=\mu, \text { where } & \\
\lambda_{i}=\mu_{n+i}, \mu_{i}=\lambda_{n+i} & (1 \leq i \leq 2 s) \\
\lambda_{i}=\mu_{i}, \lambda_{n+i}=\mu_{n+i} & (2 s<i \leq n) .
\end{array}\right)
$$

This proves $(3.2 .4)$.

Let $\mathfrak{H}_{0}$ be the group formed by the automorphs which leave un fixed. By (3.2.4), 


$$
\mathfrak{U}=\left(\mathscr{C} \mathscr{T}_{1}^{+}\right) \mathfrak{H}_{0} .
$$

Assuming that $n \neq 3$, we now prove that $\mathfrak{A}=\mathscr{C} \mathscr{T}_{1}^{+}$by showing that

$$
\mathfrak{H}_{0} \subset \mathscr{E}_{1}^{+}
$$

We consider three cases.

(a) $n=1 . \quad M=\left[ \pm \mathbf{e}_{0}, \pm \mathbf{e}_{1}\right]$, so that $\mathfrak{A}$ consists of the 8 symmetries of the square. $O_{1}^{+}$is the identity group, so that $\mathscr{C} \mathscr{T}_{1}^{+}$consists of the 8 linear transformations (2.2.2). Hence $\mathfrak{A}=\mathscr{C} \mathscr{T}_{1}^{+}$.

(b) $n=2$. The elements of $\mathfrak{U}_{0} \cap \mathscr{C} \mathscr{F}_{1}^{+}$are the 48 linear transformations (2.2.2) such that $\mathbf{t}=\mathbf{0}, g(\mathbf{0})=0$. On the other hand, $\mathfrak{A}_{0}$ permutes the elements of $M$ orthogonal to uo, viz.,

$\pm 2 \mathbf{e}_{\mathrm{a}}, \pm 2 \mathbf{e}_{\mathrm{b}}, \pm 2 \mathbf{e}_{\mathbf{c}}(\mathbf{a}, \mathbf{b}, \mathbf{c}$ the non-zero elements of $V)$, so that the order of $\mathfrak{A}_{0}$ is at most 48. Hence $\mathfrak{A}_{0}=\mathfrak{A}_{0} \cap \mathscr{C} \mathscr{T}_{1}^{+} \subset \mathscr{C} \mathscr{T}_{1}^{+}$.

(c) $n \geq 4$. We call $C_{2}$ the carrier of the vector (3.2.2). Let $N_{s}$ denote the set of vectors in $M_{s}$ whose carriers are subspaces, $N$ the union of all the $N_{s}$. If $\mathbf{v} \in M$, we have

$$
\left.\begin{array}{cr}
0 & (\mathbf{v} \notin N) \\
\mathbf{u}_{0} \cdot \mathbf{v}=2^{2\left[\frac{1}{2} n\right]-s}\left(\mathbf{v} \in N_{s}\right)
\end{array}\right)
$$

so that $\mathfrak{A}_{0}$ permutes the elements of each $N_{s}$.

Suppose now that

$$
X \in \mathfrak{A}_{0}, \mathbf{v}_{i} \in N_{1}, X \mathbf{v}_{i}=\mathbf{w}_{i} \quad(i=1,2, \ldots),
$$

and let $V_{2}^{i}, W_{2}^{i}$ be the (2-dimensional) carriers of $\mathbf{v}_{i}, \mathbf{w}_{i}$ respectively. Since $2^{2-2\left[\frac{n}{2}\right]} \mathbf{v}_{i} \circ \mathbf{v}_{j} \equiv 0$ or $1(\bmod 2)$ according as $V_{2}^{i} \cap V_{2}^{j} \neq(0)$ or $=(0)$, it follows that

$$
V_{2}^{i} \cap V_{2}^{j}=(0) \text { if, and only if, } W_{2}^{i} \cap W_{2}^{j}=(0) .
$$

Now, since $n>3$, a 2-dimensional subspace is uniquely determined by the set of 2-dimensional subspaces which meet it in the zero subspace $(0)$. Therefore, by $(3.2 .6)$,

$$
V_{2}^{i}=V_{2}^{j} \text { if, and only if, } W_{2}^{i}=W_{2}^{j}
$$

Thus, $X$ maps the set of elements of $N$ with fixed carrier $V_{2}$ onto the set of elements of $N$ with a fixed carrier $W_{2}$ which depends only on $V_{2}$. 
It now follows from case (b) that $X$ has the form

$$
X \mathbf{e}_{\alpha}=(-1)^{g(\alpha)} \mathbf{e}_{\pi(\alpha)},
$$

where $\pi$ is a mapping of $V$ onto itself whose restriction to each $V_{2}$ is a nondegenerate linear mapping into $V$. It follows that $\pi$ is a non-singular linear transformation on $V$. Also, since $[V]$ is a vector (3.2.2) or (3.2.3), $X[V]$ has the form $[V ; f]$ for some function $f$ of degree $\leq 2$. Hence $g$ has degree $\leq 2$ and so, by (2.2.2), $X \in \mathscr{C} \mathscr{T}_{1}^{+}$. This proves (3.2.5).

We mention briefly the case $n=3$. U has a subgroup isomorphic to $\left[3^{4,2,1}\right]$ (Coxeter and Moser [5], §9.4). On the other hand, an argument on the lines of (c) above shows that $\mathfrak{I}$ and $\left[3^{4,2,1}\right]$ have the same order. Hence $\mathfrak{Y} \cong\left[3^{4,2,1}\right]$.

\section{Lattices of dimension $(p-1) p^{n}$}

We pass now to the case $p>2$. Given the relation of $\mathscr{C} \mathscr{T}_{1}^{+}$to $A^{(1)}$, and the similarity in form between the elements of $\mathscr{C} \mathscr{T}_{1}^{+}$and $\mathscr{C} \mathscr{T}$, it becomes clear how to generalize $A^{(1)}$ and $A^{(2)}$. The definitions, and several alternative characterizations, are given in $\S 4.1$. The " $I$-adic" characterization is of central importance and greatly simplifies the determination of the relative minima and minimal vectors. A real metric, which turns $\Lambda^{(1)}, \Lambda^{(2)}$ into $(p-1) p^{n}$-dimensional real lattices in the usual sense, is introduced in $\S 4.2$. With these preparations the main lattice properties follow fairly easily, though the anomalous cases $p=3,5$ need some further detailed consideration.

Notation. We write

$$
\begin{aligned}
& C_{r} \mid=\theta^{n-r}\left[C_{r}\right]=\theta^{n-r} \sum_{\alpha \in C_{r}} \mathbf{e}_{\alpha}, \\
& C_{r} ; f \mid=\theta^{n-r}\left[C_{r} ; f\right]=\theta^{n-r} \sum_{\alpha \in C_{r}} \omega^{f(a)} \mathbf{e}_{\alpha}, \\
& \mathbf{u}_{\alpha}=\theta^{n} \mathbf{e}_{\alpha}
\end{aligned}
$$

where $\theta$ is the Gauss sum (2.2.4).

We write $\Pi=\omega-1$. The principal ideal $\Pi \Omega$ is prime and $\Pi^{\frac{1}{2}(p-1)} \Omega=\theta \Omega$ $=\bar{\theta} \Omega, \Pi^{p-1} \Omega=p \Omega$. The $p$ elements of the residue class ring $\Omega / \Pi \Omega$ are represented by the rational integers $0,1, \ldots, p-1$.

If $\lambda \in P$, the norm and trace of $\lambda$ relative to $R_{0}$ are denoted by $N(\lambda), \operatorname{tr} \lambda$. 4.1. The principal lattices. We define $A^{(2)}$ as the set of $\mathbf{x} \in \Gamma$ such that $X \mathbf{x} \in \Gamma$ 
for all $X \in \mathscr{C} \mathscr{T}$; in other words, it is the largest set of integral vectors invariant (as a whole) under $\mathscr{C} \mathscr{T}$. By (2.2.3), $\theta^{n} I \subset \Lambda^{(2)}$. Therefore, by lemma 2.3.1, $A^{(2)}$ is an $\Omega$-lattice.

We define $\Lambda^{(1)}$ as the dual of $\Lambda^{(2)}$ modulo $\theta^{n}$. It is an $\Omega$-lattice such that $\theta^{n} \Gamma \subset \Lambda^{(1)} \subset \Gamma$. By the argument of $\S 3.2$, every unitary transformation which leaves $A^{(2)}$ invariant also leaves $A^{(1)}$ invariant. In particular, $A^{(1)}$ is invariant under $\mathscr{C} \mathscr{T}$. Hence, by the definition of $\Lambda^{(2)}, \Lambda^{(1)} \subset \Lambda^{(2)}$.

The following is an alternative characterization of $\Lambda^{(1)}$. Consider the $\Omega$ lattice, say $A$, formed by the integral linear combinations of the vectors

$$
\left|C_{r} ; f\right| \text {, }
$$

where $C_{r}$ runs over all cosets in $V, f$ over all functions on $C_{r}$ of degree $\leq 2$. The vectors (4.1.1) are, apart from sign, the vectors $X \mathbf{u}_{\alpha}$, where $\alpha$ runs over $V, X$ over $\mathscr{C} \mathscr{T}($ see $(2.2 .3)$ and $(2.2 .5))$. Since

$$
\left(X^{-1} \mathbf{u}_{a}\right) \cdot \mathbf{x}= \pm \theta^{n} \mathbf{y}_{\alpha}
$$

where $\mathbf{y}=X \mathbf{x}$, it follows that $\Lambda$ is dual to $A^{(2)}$ modulo $\theta^{n}$. Therefore $A=\Lambda^{(1)}$.

For the remaining characterizations of $A^{(1)}, A^{(2)}$, some preparations are necessary. We define the product of two vectors by

$$
\left(x_{\alpha}\right)\left(y_{\alpha}\right)=\left(x_{\alpha} y_{\alpha}\right) \text {. }
$$

Under this product, $\Gamma$ becomes a commutative algebra over the ring $\Omega$, with unit element $\mathbf{1}=[V]$. A polynomial in the elements $\mathbf{X}, \mathbf{Y}, \ldots$ of $\Gamma$ means a sum

$$
\sum_{\lambda, \mu, \ldots, \geq_{0}} a_{\lambda \mu \ldots} \mathbf{X}^{\lambda} \mathbf{Y}^{\mu} \cdots
$$

of monomials with coefficients in 2 . The subalgebra of $I$ generated by $\mathbf{X}, \mathbf{Y}, \ldots$ means the smallest subalgebra of $\Gamma$ which contains these elements; it consists of the polynomials in $\mathbf{X}, \mathbf{Y}, \ldots$ with zero constant term $a_{00} \ldots 1$.

If $\alpha \in G F(p)$, let $\alpha^{\prime}$ denote that rational integer in the interval $[0, p-1]$ which represents $\alpha$. Write

$$
\mathbf{A}_{i}=\sum_{\left(\alpha_{1}, \ldots, \alpha_{n}\right) \in V} \alpha_{i}^{\prime} \mathbf{e}_{\left(\alpha_{1}, \ldots, \alpha_{n}\right)} \quad(i=1, \ldots, n) .
$$

Then each $\mathrm{x} \in \Gamma$ has a unique $\Pi$-adic expansion

$$
\mathbf{x} \sim \sum_{i=0}^{\infty} Q_{i}\left(\mathbf{A}_{1}, \ldots, \mathbf{A}_{n}\right) \Pi^{i},
$$


where the $Q_{i}$ are standard ${ }^{11}$ polynomials with coefficients in $[0, p-1]$. (4.1.2) means that $\mathbf{x} \equiv \sum_{0}^{k-1} Q_{i} \Pi^{i}\left(\bmod \Pi^{k} \Gamma\right)$ for all $k$.

To prove our assertion, we consider the congruences

$$
\sum_{\lambda_{1}, \ldots, \lambda_{n}=0}^{1-1} q_{\lambda_{1} \ldots \lambda_{n}} \alpha_{1}^{\prime \lambda_{1}} \cdots \alpha_{n}^{\prime \lambda_{n}} \equiv x_{\alpha}\left(\bmod \Pi^{k}\right)
$$

where $\alpha$ runs over $V$. This is a system of $p^{n}$ linear equations for the $p^{n}$ variables $q_{\lambda_{1} \ldots \lambda_{n}}$ in the residue class ring $\Omega / \Pi^{k} \Omega$. Since the determinant of the system is a power of $\prod_{i<j}\left(\alpha_{i}^{\prime}-\alpha_{j}^{\prime}\right)$ and so a unit of $\Omega / \Pi^{k} \Omega$, the solution is unique. Thus, $\mathbf{x} \equiv Q\left(\mathbf{A}_{1}, \ldots, \mathbf{A}_{n}\right)\left(\bmod \Pi^{k} \Gamma\right)$, where $Q$ is a standard polynomial over $\lambda$, whose coefficients are unique modulo $\Pi^{k}$. Replacing the coefficients of $Q$ by their $\Pi$-adic representations, we get the required unique representation $\mathrm{x} \equiv \sum_{0}^{k-1} Q_{i} \Pi^{i}\left(\bmod \Pi^{k} \Gamma\right)$.

If $x_{\alpha}$ is a function of $\alpha_{i_{1}}, \ldots, \alpha_{i_{t}}$ only, each $Q_{i}$ is a polynomial in $\mathbf{A}_{i_{1}, \ldots}, \mathbf{A}_{i_{t}}$ only. This follows from the $\Pi$-adic expansion of the $p^{t}$-dimensional vector $y_{\left(\alpha_{i_{1}}, \ldots, \alpha_{i t}\right)}$ $=x_{\alpha}$.

We return now to $\Lambda^{(1)}, \Lambda^{(2)}$. We first prove that $\Lambda^{(1)}$ is the subalgebra of $\Gamma$ generated by the vectors

$$
|V ; f|,\left|C_{n-1}\right|
$$

where $f$ runs over all functions of degree $\leq 2, C_{n-1}$ over all $(n-1)$-dimensional cosets.

Consider the product $S=\left|C_{r} ; f\right|\left|C_{s} ; g\right|$, where $f, g$ have degree $\leq 2$. If $C_{r} \cap C_{s}$ is empty, $S=0$. If not, $C_{r} \cap C_{s}=C_{t}$, where $n \geq \operatorname{dim}\left(C_{r}+C_{s}\right)=r+s-t$. Hence $S=\theta^{n+t-r-s}\left|C_{t} ; f+g\right| \in \Lambda^{(1)}$. This proves that $\Lambda^{(1)}$ is a subalgebra. The elements (4.1.3) are generators because $\left|C_{r} ; f\right|=|V ; f| \prod_{i=1}^{r}\left|C_{n-1}^{(i)}\right|$ for any $(n-r) C_{n-1}^{(i)}$ 's with meet $C_{r}$.

Let $L^{(1)}$ denote the set of $\mathrm{x} \in \Gamma$ such that, in the $\Pi$-adic expansion (3.1.2),

$$
\text { degree } Q_{i} \leq 2 i \quad(i=0,1, \ldots) .
$$

Since (4.1.4) places no restriction on $Q_{i}$ when $i \geq \frac{1}{2} \boldsymbol{n}(\boldsymbol{p}-1)$, we have

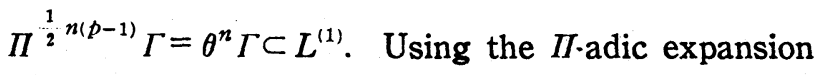

11) i.e., the degree of $Q_{i}$ in each variable is $\langle p$; cf $\$ 2.1$. 


$$
\mathbf{A}_{i}^{p}=\mathbf{A}_{i}+\Pi^{p-1} Q_{p-1}\left(\mathbf{A}_{i}\right)+\cdots
$$

of $\mathbf{A}_{i}^{p}$, it can be verified that products and $\Omega$-linear combinations of elements of $L^{(1)}$ are again in $L^{(1)}$; therefore $L^{(1)}$ is a subalgebra of $\Gamma$. We prove now that

$$
L^{(1)}=\Lambda^{(1)} \text {. }
$$

Notation. If $S$ is a subset or element of $\Gamma, S^{*}$ denotes the corresponding subset or element in the factor algebra $\Gamma^{*}=\Gamma / \theta^{n} \Gamma$. The elements of $\Gamma^{*}$ are regarded as vectors over the residue class ring $\Omega^{*}=\Omega / \theta^{n} \Omega . \mathscr{P}\left(\mathbf{X}^{*}, \ldots\right)$ denotes the subalgebra of $I^{*}$ generated by $\mathbf{x}^{*}, \ldots$

Proof of (4.1.5) Considering the monomials in the $\Pi$-adic expansion, we see that $L^{(1) *}$ is generated by the $n^{2}+n+1$ elements

$$
\mathbf{1}^{*}, \Pi \mathbf{A}_{i}^{*}, \Pi \mathbf{A}_{i}^{*} \mathbf{A}_{j}^{*} .
$$

Since every $|V ; f|$ in (4.1.3) is a polynomial in the $n^{2}+n+1$ vectors

$$
1, \mathbf{a}_{i}=\left|V ; \alpha_{i}\right|-1, \mathbf{a}_{i j}=\left|V ; \alpha_{i} \alpha_{j}\right|-1,
$$

$\Lambda^{(11 *}$ is generated by the corresponding elements

$$
\mathbf{1}^{*}, \mathbf{a}_{i}^{*}, \mathbf{a}_{i j}^{*}
$$

and the vectors

$$
\left|C_{n-1}\right|^{*}
$$

We prove $(4.1 .5)$ by showing that

(A) the elements (4.1.6) and (4.1.7) can be expressed in terms of one another;

(B) the elements (4.1.8) can be expressed in terms of the elements (4.1.6).

The proof of $(\mathrm{A})$ is simplified by the following lemma, whose easy proof is omitted.

Lemma. Let $S$ be a subalgebra of $\Gamma^{*}$ and $\mathrm{x}^{*}, \ldots$ elements of $S$. Then $S=\mathscr{P}\left(\mathbf{X}^{*}, \ldots\right)$ if, and only if, $S / \Pi S=\mathscr{P}\left(\mathbf{X}^{*}+\Pi S, \ldots\right)$.

Consider now the elements $\mathbf{a}_{i}^{*}$. The $\alpha$-th coordinate of $\mathbf{a}_{i}$ is

$$
\omega^{\alpha_{i}}-1=(1+\Pi)^{\alpha_{i}^{\prime}}-1=\sum_{j=1}^{\alpha_{i}^{\prime}}\left(\begin{array}{c}
\alpha^{\prime} \\
j^{i}
\end{array}\right) \Pi^{j}
$$

It follows that 


$$
\mathbf{a}_{i}^{*}=\sum_{j=1}^{p-1}(j !)^{-1} \prod_{k=0}^{j-1}\left(\Pi \mathbf{A}_{i}^{*}-k \Pi\right)+\Pi^{p} \mathbf{b}_{i}^{*}
$$

where ()$^{-1}$ denotes the inverse in $\Omega^{*}$ and $b_{i}$ is a vector in $\Gamma$ whose $\boldsymbol{\alpha}$-th coordinate depends only on $\alpha_{i}$. From the $I I$-adic expansion of $\mathbf{b}_{i}$, we deduce that $\Pi^{p} \mathbf{b}_{i}^{*} \in \Pi \mathscr{P}\left(\Pi \mathbf{A}_{i}^{*}\right)$. It follows that $\mathbf{a}_{i}^{*} \in \mathscr{P}\left(\Pi \mathbf{A}_{i}^{*}\right)$. Further, since

$$
\mathbf{a}_{i}^{*} \equiv \sum_{j=1}^{p-1}(j !)^{-1}\left(\Pi \mathbf{A}_{i}^{*}\right)^{j} \quad\left(\bmod I I \mathcal{I}\left(\Pi \mathbf{A}_{i}^{*}\right)\right),
$$

we have

$$
\Pi \mathbf{A}_{i}^{*} \equiv \sum_{j=1}^{\nu-1}(-1)^{j-1} j^{-1}\left(\mathbf{a}_{i}^{*}\right)^{j}\left(\bmod \Pi \mathscr{P}\left(\Pi \mathbf{A}_{i}^{*}\right)\right)
$$

whence, by the lemma, $\mathscr{P}\left(I \mathbf{A}_{i}^{*}\right)=\mathscr{P}\left(\mathbf{a}_{i}^{*}\right)$.

By similar arguments, we get

$$
\begin{gathered}
\mathscr{P}\left(\Pi \mathbf{A}_{i}^{*}, \Pi \mathbf{A}_{i}^{* 2}\right)=\mathscr{P}\left(\mathbf{a}_{i}^{*}, \mathbf{a}_{i i}^{*}\right), \\
\mathscr{P}\left(\Pi \mathbf{A}_{i}^{*}, \Pi \mathbf{A}_{j}^{*}, \Pi \mathbf{A}_{i}^{* 2}, \Pi \mathbf{A}_{j}^{* 2}, \Pi \mathbf{A}_{i}^{*} \mathbf{A}_{j}^{*}\right)=\mathscr{P}\left(\mathbf{a}_{i}^{*}, \mathbf{a}_{j}^{*}, \mathbf{a}_{i i}^{*}, \mathbf{a}_{j j}^{*}, \mathbf{a}_{i j}^{*}\right),
\end{gathered}
$$

whence (A) follows.

By (A), and because of the symmetry of the set of vectors $|V ; f|$ with respect to index transformations $\alpha \rightarrow \alpha D+t$, it is sufficient to prove (B) when $C_{n-1}$ is the particular coset defined by the equation $\alpha_{1}=0$. Now the $\pi$-adic expansion shows that $\left[C_{n-1}\right]^{*}$ is a polynomial of degree $<p$ in $\mathbf{A}_{1}^{*}$. It follows that $\Pi^{{ }^{1}{ }^{(p-1)}}\left[C_{n-1}\right]^{*} \in \mathscr{\rho}\left(\Pi \mathbf{A}_{1}^{*}, \Pi \mathbf{A}_{1}^{* 2}\right)$ and therefore, since $\Pi^{\frac{1}{2}(p-1)} \Omega=\theta \Omega$, that $\left|\boldsymbol{C}_{n-1}\right|^{*} \in \mathscr{P}\left(\Pi \mathbf{A}_{1}^{*}, \Pi \mathbf{A}_{1}^{* 2}\right)$. This proves (B) and (4.1.5).

There is a similar $\Pi$-adic characterization of $A^{(2)}$. Let $L^{(2)}$ denote the set of $\mathbf{x} \in \Gamma$ such that

$$
\text { degree } Q_{i} \leq 2 i+1 \quad(i=0,1, \ldots)
$$

then

$$
L^{(2)}=A^{(2)}
$$

This is proved by showing that

(C) $\left|\Gamma: L^{(1)}\right|\left|\Gamma: L^{(2)}\right|=\left|\Gamma: \theta^{n} \Gamma\right|$,

(D) $\mathbf{x} \cdot \mathbf{y} \equiv 0\left(\bmod \theta^{n}\right)$ whenver $\mathbf{x} \in L^{(1)}, \mathbf{y} \in L^{(2)}$.

(C), (D) imply that $L^{(1)}, L^{(2)}$ are dual modulo $\theta^{n}$ and thus that $L^{(2)}=A^{(2)}$, as required. 
It is an easy combinatorial problem to show that

$$
\left|L^{(1)}: \theta^{n} \Gamma\right|=p^{k_{2}},\left|L^{(2)}: \theta^{n} \Gamma\right|=p^{k_{1}},
$$

where

(4. 1.11) $\quad k_{1}=\frac{1}{4}\left[n(p-1)+\left(p^{n}-1\right)\right], k_{2}=\frac{1}{4}\left[n(p-1)-\left(p^{n}-1\right)\right]$.

Since $\left|\Gamma: \theta^{n} \Gamma\right|=p^{\frac{1}{2} n(p-1) p^{n}}$ and $k_{1}+k_{2}=\frac{1}{2} n(p-1) p^{n}$, we get $(\mathrm{C})$ and

$$
\left|\Gamma: L^{(i)}\right|=p^{k_{i}} \quad(i=1,2) .
$$

It is sufficient to prove (D) when

$$
\mathbf{x}=\Pi^{\lambda} \mathbf{A}_{1}^{\lambda_{1}} \cdots \mathbf{A}_{n}^{\lambda_{n}}, \mathbf{y}=\Pi^{\mu} \mathbf{A}_{1}^{\mu_{1}} \cdots \mathbf{A}_{n}^{\mu_{n}},
$$

where

$$
\begin{array}{ll}
0 \leq \lambda_{i}<p, & 0 \leq \mu_{i}<p \\
\sum \lambda_{i} \leq 2 \lambda, & \sum \mu_{i} \leq 2 \mu+1 .
\end{array}
$$

We may suppose that $\lambda+\mu<\frac{1}{2} n(p-1)$, for $(D)$ is obvious otherwise. Let $k$ be the integral part of $(p-1)^{-1} \sum\left(\lambda_{i}+\mu_{i}\right)$. Since

$$
k(p-1) \leq \sum\left(\lambda_{i}+\mu_{i}\right) \leq 2 \lambda+2 \mu+1<n(p-1),
$$

we have

$$
k<n, \frac{1}{2} k(p-1)<\lambda+\mu .
$$

Let $r$ be the number of indices $i$ such that $\lambda_{i}+\mu_{i} \neq(p-1)$. Clearly $(n-r)(p-1) \leq \sum\left(\lambda_{i}+\mu_{i}\right)$, so that

$$
r \geq n-k \text {. }
$$

Now

$$
\begin{aligned}
\mathbf{x} \cdot \mathbf{y} & =\bar{\Pi}^{\lambda} \Pi^{\mu} \sum_{a \in I^{\prime}} \alpha_{1}^{\prime \lambda_{1}+\mu_{1}} \cdots \alpha_{n}^{\prime \lambda_{n}+\mu_{n}} \\
& =\bar{\Pi}^{\lambda} \Pi^{\mu} s_{\lambda_{1}+\mu_{1}} \cdots s_{\lambda_{n}+\mu_{n}},
\end{aligned}
$$

where

$$
s_{0}=p, s_{k}=1^{k}+2^{k}+\cdots+(p-1)^{k} \quad(k>0) .
$$

Since, for $k>0, s_{k} \equiv-1$ or $0(\bmod p)$ according as $(p-1) \mid k$ or not, we have

$$
\mathbf{x} \cdot \mathbf{y} \equiv 0\left(\bmod \Pi^{\lambda+\mu+r(p-1)}\right) \text {. }
$$

(D) now follows from (4.1.13), (4.1.14). This proves (4.1.10).

4.2. The real metric. $E$, as defined in $\S 2.2$, is a $p^{n}$-dimensional metric space 
over $P$. We now describe a natural way of defining it as a $(p-1) p^{n}$-dimensional metric space over $R_{\mathbf{0}}$.

Consider first the degenerate case $n=0$, where $E=P$ and $\Gamma=\Omega . \quad P$ is a $(p-1)$-dimensional vector space over $R_{0}$. It becomes a metric space over $R_{0}$ if we define the real scalar product by

$$
\lambda * \mu=\operatorname{tr} \bar{\lambda} \mu .
$$

Since the Galois group $G$ of $P$ over $R_{0}$ is abelian,

$$
\lambda * \lambda=\sum_{\sigma \in G}(\bar{\lambda} \lambda)^{\sigma}=\sum_{\sigma \in G} \bar{\lambda}^{\sigma} \lambda^{\sigma}
$$

whence $\lambda * \mu$ is positive definite.

$\Omega$ becomes a $(p-1)$-dimensional lattice in the usual sense. The roots of unity $\omega^{i}(1 \leq i \leq p-1)$ form a lattice basis. By evaluating $\operatorname{det}\left(\operatorname{tr} \omega^{i-j}\right)$, we get

$$
D(\Omega)=p^{p-2} \text {. }
$$

The inequality of the arithmetic and geometric means shows that $(p-1)|N(\lambda)|^{2}$ $\leq \lambda * \lambda$, with equality if, and only if, all conjugates of $\lambda$ have the same modulus. Hence the minimal vectors of $\Omega$ are the roots of unity $\pm \omega^{i}$ and

$$
M(\Omega)=p-1
$$

In the general case, $E$ is a vector space over $R_{0}$ of dimension $N=(p-1) p^{n}$, and we define the real metric $\mathbf{x} * \mathbf{y}$ by

$$
\left(x_{\alpha}\right) *\left(y_{\alpha}\right)=\sum_{\alpha \in V} x_{\alpha} * y_{\alpha}=\operatorname{tr}\left(x_{\alpha}\right) \cdot\left(y_{\alpha}\right) .
$$

$\Gamma$ is an $N$-dimensional lattice with basis $\omega^{i} \mathbf{e}_{\alpha}(\alpha \in V, 1 \leq i \leq p-1) . \quad$ By (4.2.3), (4.2.4),

$$
D(\Gamma)=p^{(p-2) p^{n}}, M(\Gamma)=p-1
$$

Hence, by (4.1.12),

$$
D\left(\Lambda^{(i)}\right)=p^{(p-2) p^{n+2} k_{i}} \quad(i=1,2),
$$

where $k_{i}$ is given by (4.1.11).

The following results are noted for future reference. Let $\lambda \in \Omega$ and let $k$ be the rational integer in $[0, p-1]$ such that $\lambda \equiv k(\bmod \Pi)$. By $(4.2 .2), \lambda * \lambda$ is even and $\equiv-k^{2}(\bmod p)$. Hence 


$$
\left.\begin{array}{lll}
\geq 2 p & \text { if } k=0, \lambda \neq 0, \\
\lambda * \lambda & \geq p-1 & \text { if } k=1, p-1 \\
& \geq p+1 & \text { otherwise. }
\end{array}\right)
$$

In particular, when $p=5$ and $k=2,3$, equality holds if, and only if, $\lambda$ is one of the 20 numbers $\pm\left(\omega^{i}+\omega^{j}\right) \quad(0 \leq i<j \leq 4)$.

4.3. Minima, minimal vectors. We now determine the minimal vectors of $A^{(1)}, A^{(2)}$. Let $\mathbf{x}=\Pi^{s} \mathbf{y} \in A^{(2)}(s \geq 0)$, where $\mathbf{y} \in \Lambda^{(2)}$ but $\Pi^{-1} \mathbf{y} \notin \Lambda^{(2)}$. By the original definition of $A^{(2)}$, there is an $X \in \mathscr{C} \mathscr{T}$ such that $\mathrm{z}=X \mathbf{y} \notin \Pi \Gamma$. Let

$$
\mathrm{z} \sim \sum_{0}^{\infty} Q_{i}\left(\mathbf{A}_{1}, \ldots, \mathbf{A}_{n}\right) \Pi^{i}
$$

and let

$$
\widetilde{Q}_{i}\left(\alpha_{1}, \ldots, \alpha_{n}\right)=\sum a_{\lambda_{1} \ldots \lambda_{n}} \alpha_{1}^{\lambda_{1}} \ldots \alpha_{n}{ }^{\lambda_{n}} \quad(i=0,1, \ldots)
$$

be the unique standard polynomial over $G F(p)$ such that

$$
\sum a_{\lambda_{1} \ldots \lambda_{n}}^{\prime} \alpha_{1}^{\prime \lambda_{1}} \ldots \alpha_{n}^{\prime \lambda_{n}}=Q_{i}\left(\alpha_{1}^{\prime}, \ldots \alpha_{n}^{\prime}\right) .
$$

If $Y$ is the transformation (2.2.5), $Y \mathbf{w}=\mathbf{z}$ and

$$
\mathbf{w} \sim \sum_{0}^{\infty} R_{i}\left(\mathbf{A}_{1}, \ldots, \mathbf{A}_{n}\right) \Pi^{i},
$$

then

$$
\widetilde{R}_{0}(\alpha)=\widetilde{Q}_{0}(\alpha D+\mathrm{t}), \widetilde{R}_{1}(\alpha)=\widetilde{Q}_{1}(\alpha D+\mathrm{t})-g(\alpha) \widetilde{Q}_{0}(\alpha D+\mathrm{t}) .
$$

After applying such a transformation we may therefore suppose that either (a) $Q_{0}=k 1(k \neq 0)$ or (b) $Q_{0}=\mathbf{A}_{1}$. Notice that $\mathrm{z} \notin \Lambda^{(1)}$ in case (b), by (4.1.4).

Case $(a)$. Every coordinate $z_{\alpha}$ is non-zero, whence by (4.2.4),

$$
\mathbf{x} * \mathbf{x}=\left(\Pi^{s} \mathbf{z}\right) *\left(\Pi^{s} \mathbf{z}\right) \geq(p-1) p^{n}
$$

with equality if, and only if, $s=0$ and $z_{\alpha}$ is a $(2 p)$-th root of unity for each $\alpha$. Suppose that equality holds. After replacing $\mathrm{z}$ by $-\mathrm{z}$ if necessary, we have $k=1$ and $\mathrm{z}=[V ; f]$ for some standard polynomial $f\left(\alpha_{1}, \ldots, \alpha_{n}\right)$. Then, expanding $z_{\alpha}=(1+\pi)^{j}$ by the binomial theorem, we get

$$
\widetilde{Q}_{i}\left(\alpha_{1}, \ldots, \alpha_{n}\right)=\left(\begin{array}{c}
f\left(\alpha_{1}, \ldots, \alpha_{n}\right) \\
i
\end{array}\right) \quad(i=1, \ldots, p-2) .
$$

Therefore, since $\widetilde{Q}_{1}$ and $f$ are standard, $\widetilde{Q}_{1}\left(\xi_{1}, \ldots, \xi_{n}\right)=f\left(\xi_{1}, \ldots, \xi_{n}\right)$ identically in independent variables $\xi_{i}$. After applying a suitable transforma- 
tion (2.2.5), we may suppose that all terms in $f$ of degree $\leq 2$ are zero.

Suppose now that $\mathbf{x} \in \Lambda^{(1)}$. Then $\mathbf{z} \in A^{(1)}$ and so, by (4.1.4), the degree of $\widetilde{Q}_{1} \leq 2$. Hence $f=0$ and $\mathbf{z}=[V]$. Suppose secondly that $\mathbf{x} \in A^{(2)}, \mathbf{x} \notin A^{(1)}$. Then, by (4.1.9), the degree of $\widetilde{Q}_{1} \leq 3$, so that $f$ is a homogeneous cubic. If $p \geq 7$,

$$
\widetilde{Q}_{2}\left(\xi_{1}, \ldots, \xi_{n}\right)=\left(\begin{array}{l}
f\left(\xi_{1}, \ldots, \xi_{n}\right) \\
2
\end{array}\right)
$$

since the latter is standard. This is impossible because $\left(\begin{array}{l}f \\ 2\end{array}\right)$ has degree $6, \widetilde{Q}_{2}$ degree $\leq 5$. Hence $p=3$ or 5 . We need not consider the case $p=3$, because case (b) shows that $\mathrm{z} * \mathrm{z}>M^{(2)}=4.3^{n-1}$. Suppose then that $p=5$. After a suitable transformation $(2.2 .5)$, we may suppose that

$$
f\left(\alpha_{1}, \ldots, \alpha_{n}\right)=\alpha_{1}^{3}+\alpha_{1} q\left(\alpha_{2}, \ldots, \alpha_{n}\right)+r\left(\alpha_{2}, \ldots, \alpha_{n}\right),
$$

where $q, r$ are homogeneous of degrees 2,3 respectively. Then, after reduction to standard form, $\left(\begin{array}{l}f \\ 2\end{array}\right)$ contains the sextic terms $\alpha_{1}^{4} q+\alpha_{1}^{3} r$, so that $q=r=0$. Thus $\mathrm{z}=\left[V ; \alpha_{1}^{3}\right]$. It is easy to see that this vector is actually in $A^{(2)}$.

Case $(b)$. We have $z_{\alpha} \equiv \alpha_{1}^{\prime}(\bmod I)$, whence, by (4.2.8),

$$
\begin{gathered}
\mathbf{x} * \mathbf{x} \geq(p-1) p^{n} \times 2 p=2(p-1) p^{n} \quad \text { if } s>0 \\
\mathbf{x} * \mathbf{x} \geq(p-3) p^{n-1} \times(p+1)+2 p^{n-1} \times(p-1)=\left(p^{2}-5\right) p^{n-1} \quad \text { if } s=0 .
\end{gathered}
$$

By case (a), $\mathbf{x}$ cannot be a minimal vector of $A^{(2)}$ unless $s=0$ and $p=3$ or $\overline{5}$. Notice that in these cases $\mathrm{x} \notin \Lambda^{(1)}$ because $s=0$ and $\mathrm{z} \notin \Lambda^{(1)}$. Let $C^{\lambda}$ denote the $(n-1)$-dimensional coset in $V$ defined by the equation $\alpha_{1}=\lambda$. Suppose first that $p=3$ and $\mathbf{x} * \mathbf{x}=\left(3^{2}-5\right) 3^{n-1}=4.3^{n-1}$. By (4.2.4), and since $Q_{0}=\mathbf{A}_{1}$,

$$
\mathrm{z}=\left[C^{1} ; f\right]-\left[C^{-1} ; g\right]
$$

where $f, g$ are standard polynomials in $\alpha_{2}, \ldots, \alpha_{n}$. Using the equations

$$
\left|C^{1}\right|=2 \mathbf{A}_{1}-\mathbf{A}_{1}^{2},\left|C^{-1}\right|=\frac{1}{2}\left(\mathbf{A}_{1}^{2}-\mathbf{A}_{1}\right),
$$

we get

$$
\widetilde{Q}_{1}=\alpha_{1}^{2}(g-f)-\alpha_{1}(g+f),
$$

whence the degrees of $g-f, g+f$ are $\leq 1,2$ respectively. Then the element

$$
Y \mathbf{e}_{\alpha}=\omega^{-\frac{1}{2}\left[(f+g)+\alpha_{1}\left(f-g^{\prime}\right)\right]} \mathbf{e}_{\alpha}
$$


of $\mathscr{C} \mathscr{T}$ maps z onto

$$
\left[C^{1}\right]-\left[C^{-1}\right]=\mathbf{A}_{1}+3 / 2\left(\mathbf{A}_{1}-\mathbf{A}_{1}^{2}\right) .
$$

The $\Pi$-adic expansion shows that this vector is in $A^{(2)}$.

Suppose now that $p=5$ and $\mathbf{x} * \mathbf{x}=\left(5^{2}-5\right) 5^{n-1}=4.5^{n}$. By (4.2.8), and since $Q_{0}=\mathbf{A}_{1}$,

$$
\mathbf{z}=\left[C^{1} ; f\right]-\left[C^{-1} ; g\right]+\left(\left[C^{2} ; h_{1}\right]+\left[C^{2} ; h_{2}\right]\right)-\left(\left[C^{-2} ; k_{1}\right]+\left[C^{-2} ; k_{2}\right]\right)
$$

where $f, g, \ldots$ are standard polynomials in $\alpha_{2}, \ldots, \alpha_{n}$ and neither $h_{1}-h_{2}$ nor $k_{1}-k_{2}$ assumes the value 0 . Then

$$
\begin{aligned}
\widetilde{Q}_{1}= & \alpha_{1}^{4}(-f+g-h+k)+\alpha_{1}^{3}(-f-g-2 h-2 k) \\
& +\alpha_{1}^{2}(-f+g+h-k)+\alpha_{1}(-f-g+2 h+2 k)
\end{aligned}
$$

where $h=h_{1}+h_{2}, k=k_{1}+k_{2}$. Since the degree of $\widetilde{Q}_{1} \leq 3$, the coefficient of $\alpha_{1}^{4}=0$ and those of $\alpha_{1}^{3}, \alpha_{1}^{2}, \alpha_{1}$ have degrees $\leq 0,1,2$ respectively. After applying the transformation

$$
Y \mathbf{e}_{\alpha}=\omega^{-\frac{1}{2}(f+g)-\frac{1}{2} \alpha_{1}(f-g)-c\left(\alpha_{1}^{2}-1\right)} \mathbf{e}_{\alpha},
$$

where $c$ is the constant $h+2 f+\mathrm{g}$, we have $f=g=h=k$, so that $h_{1}=-h_{2}$, $k_{1}=-k_{2}$. The next term of the $\Pi$-adic expansion now gives

$$
\widetilde{Q}_{2}=\left(\alpha_{1}^{3}-\alpha_{1}\right)\left[\left(k_{1}^{2}-h_{1}^{2}\right) \alpha_{1}-2\left(h_{1}^{2}+k_{1}^{2}\right)\right] .
$$

Since the functions $h_{1}-h_{2}=2 h_{1}$ and $k_{1}-k_{2}=2 k_{1}$ do not assume the value 0 , it follows that $h_{1}^{2}, k_{1}^{2}$ are functions of degree $\leq 2$ which can assume only the values $1,-1$. It is easy to see that every function of degree 1 or 2 assumes at least 3 values, so that $h_{1}^{2}, k_{1}^{2}$ are constants. Hence, after applying the transformation $Y \mathbf{e}_{\alpha}=-\mathbf{e}_{-\alpha}$ if necessary, $\mathrm{z}$ becomes one of the 4 vectors ${ }^{4}$

$$
\mathbf{v}_{r, s}=\left[C^{1}\right]-\left[C^{-1}\right]+\left(\omega^{r}+\omega^{-r}\right)\left[C^{2}\right]-\left(\omega^{s}+\omega^{-s}\right)\left[C^{-2}\right],
$$

where $(r, s)=(1,1),(1,2),(2,1)$ or $(2,2)$. The $\Pi$-adic expansion shows that $\mathbf{v}_{r, s} \in \Lambda^{(2)}$. The transformation

$$
Y \mathbf{e}_{\left(\lambda, \alpha_{2}, \ldots, \alpha_{n}\right)}=\theta^{-1} \sum_{\mu} \omega^{(\lambda-\mu)^{2}} \mathbf{e}_{\left(\mu, \alpha_{2}, \ldots, \alpha_{n}\right)}
$$

belongs to $\mathscr{C} \mathscr{T}$ and maps $\mathbf{v}_{12}, \mathbf{v}_{21}$ into vectors with all coordinates non-zero. Hence the minimal vector pairs $\pm\left[V ; \alpha_{1}^{3}\right], \pm \mathbf{v}_{12}, \pm \mathbf{v}_{21}$ are equivalent under $\mathscr{C} \mathscr{T}$. It can be shown, though we omit the proof, that no two of the pairs 
$\pm[V], \pm\left[V ; \alpha_{1}^{3}\right], \pm \mathbf{v}_{11}, \pm \mathbf{v}_{22}$ are equivalent under $\mathscr{C} \mathscr{T}$

We have proved

THEOREM 4.3.1. The minimal vector pairs of $A^{(i)}$ are given by (4.1.1) except when $i=2$ and $p=3$ or 5 . When $i=2, p=3$, every minimal vector pair is equivalent under $\mathscr{C} \mathscr{G}$ to $\pm\left(\left[C^{1}\right]-\left[C^{-1}\right]\right)$, where $C^{\lambda}$ is the $(n-1)$-dimensional coset defined by $\alpha_{1}=\lambda$. When $i=2, p=5$, every minimal vector pair is equivalent under $\mathscr{C} \mathscr{T}$ to one, and only one, of the pairs $\pm[V], \pm\left[V ; \alpha_{1}^{3}\right]$ and

$$
\pm\left\{\left[C^{1}\right]-\left[C^{-1}\right]+\left(\omega^{i}+\omega^{-i}\right)\left(\left[C^{2}\right]-\left[C^{-2}\right]\right)\right\} \quad(i=1,2)
$$

where $C^{\lambda}$ has the same meaning as in the case $p=3$.

THEOREM 4.3.2. The relative minima $\gamma^{(1)}, \gamma^{(2)}$ of $A^{(1)}, A^{(2)}$ are given by

$$
\begin{array}{ll}
r^{(1)}=(p-1) p^{\frac{1}{2} n-1+\frac{1}{2}(p-1)^{-1}\left(1+p^{-n}\right)} & (p \geq 3) \\
\gamma^{(2)}=(p-1) p^{\frac{1}{2} n-1+\frac{1}{2}(p-1)^{-1}\left(3-p^{-n}\right)} & (p>3) \\
\gamma^{(2)}=4.3^{\frac{1}{2} n-\frac{5}{4}-\frac{1}{4} 3^{-n}} & (p=3) .
\end{array}
$$

We can compare our forms with the original ones in $2^{n}$ variables by computing $\rho_{i}(p)=\lim _{i \rightarrow \infty} \gamma^{(i)} /\left(\frac{1}{2} N\right)^{\frac{1}{2}}$. We have:

$$
\begin{aligned}
& \rho_{1}(3) \approx 0.9, \rho_{1}(5) \approx 0.7, \ldots \\
& \rho_{2}(3)=4.3^{-\frac{5}{4}} \approx 1.01, \rho_{2}(5)=2^{15 / 8} 5^{-5 / 8} \approx 1.03, \rho_{2}(7) \approx 0.8, \ldots \\
& \lim _{p \rightarrow \infty}\left(\frac{1}{2} p\right)^{\frac{1}{2}} \rho_{i}(p)=1 \quad(i=1,2) .
\end{aligned}
$$

The lowest values of $(p-1) p^{n}$ are $6,18,20$ corresponding to $p^{n}=3,9,5$ respectively. The forms in 6 variables are the absolutely extreme and "next best" extreme. The relative minima of the forms in 18, 20 variables for $i=2$ are

$$
4 / 3^{5 / 18} \approx 2.95,4 / 5^{3 / 20} \approx 3.1
$$

These are comparable with the value $8^{\frac{1}{2}} \approx 2.8$ for the 16 -variable form of EF. 4.4. Extreme Forms. Two points can be made at once. 
(1) $1^{(i)}$ is invariant under the $R_{0}$-irreducible ${ }^{12)}$ group $\mathscr{C} \mathscr{T}$. Therefore it is eutactic (Coxeter [4], p. 402).

(2) Let $S$ be the automorphism of $P$ such that $\omega^{s}=\omega^{2}$ and let $\mathbf{a}$ be any element of $V$. Then it is easily verified that, if $p>3$, every vector (4.1.1) satisfies the quadratic relation

$$
x_{3 \mathrm{a}}^{s} x_{-3 \mathrm{a}}=x_{-\mathrm{a}}^{s} x_{5 \mathrm{a}} .
$$

Therefore, if $p>3, A^{(i)}$ cannot be perfect when the vectors (4.1.1) and their negatives are its minimal vectors; i.e., $A^{(i)}$ cannot be perfect unless $p=3$ or $p=5, i=2$. We shall see, however, that $A^{(i)}$ is perfect in a modified sense now to be defined.

Let $A$ be a sublattice of the $N$-dimensional lattice $\Gamma$. Let $G(\mathbf{x}, \mathbf{y})$ stand generically for an $R_{0}$-bilinear function defined on $E$ and with complex values. Then, according to the usual definition, $A$ is perfect if the equations

$$
G(\mathbf{m}, \mathbf{m})=0 \text { for all minimal vectors } \mathbf{m} \text { of } A \text {, }
$$

imply that

$$
G(\mathbf{x}, \mathbf{x})=0 \text { for all } \mathbf{x} \in E .
$$

We may, without loss of generality, suppose in the definition that the values of $G(\mathbf{x}, \mathbf{y})$ are in $R_{0}$.

Let now $t(\neq 0) \in G F(p)$ and let $T$ be the automorphism of $P$ such that $\omega^{T}=\omega^{t}$. We call $A t$-perfect if the implication $(4.4 .1) \Rightarrow(4.4 .2)$ holds for functions of the form

$$
G(\mathbf{x}, \mathbf{y})=\sum_{a, \beta} g_{\alpha, \beta} x_{\alpha}^{T} y_{\beta} \quad\left(g_{a, \beta} \in P\right) .
$$

Clearly, if $A$ is perfect it is $t$-perfect for all $t$. The converse is also true. In fact, let $G$ be as in the previous paragraph. Then

$$
G_{t}(\mathbf{x}, \mathbf{y})=\sum_{i, j=0}^{p-1} \omega^{-t i-j} G\left(\omega^{i} \mathbf{x}, \omega^{j} \mathbf{y}\right)
$$

has the form (4.4.3), and

$$
p^{2} G(\mathbf{x}, \mathbf{y})=\operatorname{tr}\left(\sum_{l=1}^{p-1} G_{t}(\mathbf{x}, \mathbf{y})\right)
$$

12) $\mathscr{C} \mathscr{T}$ is $R_{0}$-irreducible because it is $P$-irreducible (CGI, theorem 1) and contains the scalars $\omega I$. 
Therefore the implication $(4.4 .1) \Rightarrow(4.4 .2)$ is valid for $G$ if it is valid for each $G_{t}$.

We call $A$ P-perfect if it is 1-and $(-1)$-perfect, i.e., if it is perfect with respect to symmetric $P$-bilinear, and Hermitian, forms. We now prove

THEOREM 4.4. $A^{(i)}$ is eutactic and P-perfect. It is perfect only for $p=3$, $i=1,2$ and $p=5, i=2$.

Proof. Let $G$ be the function (4.4.3). We seek the conditions that $G(\mathbf{x}, \mathbf{x})=0$ for all vectors $\mathbf{x}=|V ; \phi|$, where $\phi$ has the form

$$
\phi(\alpha)=\sum_{i \leq j} a_{i j} \alpha_{i} \alpha_{j}+\sum_{i} a_{i} \alpha_{i}
$$

The equation $G(\mathbf{x}, \mathbf{x})=0$ gives

$$
\begin{gathered}
\sum_{\alpha, \beta} g_{\alpha, \beta} \omega^{t\langle i \alpha)+\beta(\beta)}=0 . \\
\Psi(\alpha)=\sum_{i \leq j} b_{i j} \alpha_{i} \alpha_{j}+\sum b_{i} \alpha_{i},
\end{gathered}
$$

If

we write

$$
\begin{gathered}
\phi * \Psi=\sum_{i=j} a_{i j} b_{i j}+\sum_{i} a_{i} b_{i}, \\
g_{\Psi}=\sum g_{\alpha_{\alpha}, \beta},
\end{gathered}
$$

where summation is over the $\alpha, \beta$ such that

$$
t \alpha_{i} \alpha_{j}+\beta_{i} \beta_{j}=b_{i j}, t \alpha_{i}+\beta_{i}=b_{i} \quad(\text { all } i, j)
$$

and where $g_{\Psi}=0$ when $(4.4 .5)$ has no solutions. Then $(4.4 .4)$ becomes

$$
\sum_{\forall}(\omega)^{6+n} g_{\sharp !}=0 \quad(\text { all } \phi) .
$$

The matrix $\left.(\omega)^{\omega}\right)$ is non-singular, being a direct power of the $p \times p$ matrix $\left(\omega^{i j}\right)$, and so the vector $\left(g_{4}\right)$ is zero. If $t=-1,(1.4 .5)$ has at most one solu. tion $\alpha, \beta$ whence $G(\mathbf{x}, \mathbf{y})=0$ ). If $t=1$, it has either no solution or a unique solution $\alpha, \alpha$ or exactly two solutions $\alpha, \beta$ and $\beta, \alpha$. Hence $g_{\alpha, \beta}+g_{\beta, \alpha}=0$ for all $\alpha, \beta$ and so $G(\mathbf{x}, \mathbf{x}) \equiv 0$.

We have now proved that $1^{i}$ is $P$-perfect whenever the $[V ; \phi]$ are minimal vectors, i.e., except when $p=?, i=2$. The conclusion is still true in this case.

In fact, let $\mathrm{v}_{i}=\left[C^{i} ; \phi\right](i=0,1,2)$ with $\phi$ as above and $C^{i}$ as in $\$ 1.3$. Then it is easily seen that because $G(x, x)$ vanishes for 


$$
\mathbf{v}_{0}-\omega^{i} \mathbf{v}_{1}, \mathbf{v}_{1}-\omega^{i} \mathbf{v}_{2}, \mathbf{v}_{2}-\omega^{i} \mathbf{v}_{0} \quad(i=1,2,3),
$$

it also vanishes for $\mathbf{v}_{0}+\mathbf{v}_{1}+\mathbf{v}_{2}=[V ; \phi]$. The previous argument now shows that $G(\mathbf{x}, \mathbf{x}) \equiv 0$. Hence $A^{(i)}$ is $P$-perfect in all cases.

When $p=2, A^{(i)}$ is 1 -and $(-1)$-perfect and so perfect. It remains to prove only that $A^{(2)}$ is 2 -and $(-2)$-perfect when $p=5$. This is done by applying our previous argument to $\left[V ; \alpha_{\kappa}^{3}+\phi\right]$ instead of $[V ; \phi]$. It is readily seen that the solution of (4.4.5) plus the equation $t \alpha_{\kappa}^{3}+\beta_{\kappa}^{3}=b$ is unique if either $\alpha, \alpha$ is a solution or there is a solution $\alpha, \beta$ with $\alpha_{\kappa} \neq \beta_{\kappa}$. Therefore $g_{\alpha, \beta}=0$ unless $\alpha_{\kappa}=\beta_{\kappa}$ and $\alpha \neq \beta$. Since this holds for all $\kappa, g_{\alpha, \beta}=0$ for all $\alpha, \beta$ and so $G(\mathbf{x}, \mathbf{x}) \equiv 0$. This proves the theorem.

\section{REFERENCES}

[1] Barnes, E. S. and Wall, G. E., Some extreme forms defined in terms of Abelian groups, J. Australian Math. Soc. 1 (1959), 47-63.

[2 ], [ 3 ] Bolt, Beverley, Room, T. G. and Wall, G. E., On the Clifford collineation, transform and similarity groups (I) and (II), J. Australian Math. Soc. 2 (1961), 60-96.

[ 4 ] Coxeter, H. S. M., Extreme forms, Canad. J. Maths. 3 (1951), 391-441.

[5] Coxeter, H. S. M. and Moser, W. O. J., Generators and relations for discrete groups (Springer, 1957).

[6] Dieudonné, J., La geometrie des groupes classiques (Springer, 1955).

University of Sydney

New South Wales 